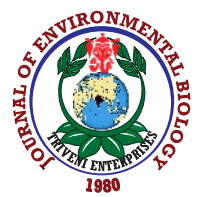

\title{
Identification of aquaporin markers in Solanum melongena for water stress response
}

\author{
R.R. Thomas* and M.K. Chandraprakash \\ Division of Social Sciences, Indian Institute of Horticultural Research, Bengaluru-560 089, India \\ *Corresponding Author Email : reenart@hotmail.com
}

\begin{abstract}
Aim: The study focused on identifying markers linked to aquaporin genes from the expressed regions of $S$. melongena using bioinformatics applications.

Methodology: The EST collections were explored for identification of aquaporin markers for water stress response using comparative analysis and inhouse developed repeat motif detection program. An algorithm was developed to generate repeat motifs which can be effectively used for collecting EST of $S$. melongena to filter the sequences having repeat motifs for further analysis.

Results: From the results generated, the 22 potential sequences with the markers were found to be associated with aquaporin proteins. The detected repeat motifs are inherent part of markers and these markers are found to be evolutionarily conserved and associated with aquaporin proteins. Hence, identifying markers for the presence of aquaporin proteins play an important role in water diffusion across cell membranes in plants.
\end{abstract}

Interpretation: Identifying aquaporin markers are useful for plant breeders for developing water stress tolerant crops during elevated temperatures. These markers are linked to water channel proteins that belong to superfamily Major Intrinsic Protein, that primarily plays an important role in conduction of water in plants.

Key words: Aquaporins, Repeat motif, Solanum melongena, Stress, Water transport

\section{Introduction}

Water is the most important limiting factor for plant growth and plays a critical role in response to environmental stresses. Aquaporins also known as water channel proteins are integral membrane pore proteins, which conduct water molecules to cells through the pores in the single file region. The presence of aquaporin protein improves the osmotic hydraulic conductivity of cell membrane (Preston et al., 1992). These are essential for the transport of water system in plants and also for drought and salt stress tolerance. These environmental stress conditions affect plant growth and yield (Cushman and Bohnert, 2000) and during drought, plants suffer from water deficit that directly affects plant water relations. A constant water flow throughout the plants and evaporation in the atmosphere is essential for carrying out their physiological activities and its growth under stress conditions. Rapid flow of water into and out of cells is mediated by the presence of aquaporins (Johansson et al., 2000). Plant aquaporins recognized as water channel proteins are membrane intrinsic proteins (21 to $34 \mathrm{kD}$ ) that belong to the Major Intrinsic Protein (MIP) superfamily (Tyerman et al., 1999). They are expressed in various membranes of plant cells and are localized

How to cite : Thomas, R.R. and M.K. Chandraprakash: Identification of aquaporin markers in Solanum melongena for water stress response. J. Environ. Biol., 41, 1570-1574 (2020). 
to the tonoplast and plasma membranes (Kapilan et al., 2018) by forming a proteinaceous pore in the membrane. High expression level of tonoplast aquaporin allows rapid flow of water through living cells (Barrieu et al., 1998).

Brinjal (Solanum melongena L.) an important vegetable crop can tolerate greater levels of drought stress (Behboudian, 1977) because of efficient mechanisms to transfer water across the plant body and to control the negative effects of drought. The genome size of brinjal is $1127 \mathrm{Mb}$ and it was predicted that there could be 85,446 genes including pseudo genes in the genome (Hirakawa et al., 2014). In Arabidopsis thaliana, 35 genes for aquaporins (Chrispeels et al., 1999, Johanson et al., 2001), 31 in maize (Chaumont et al., 2001), 33 in rice (Sakurai et al., 2005), 72 in soybean (Zhang et al., 2013), and 47 in tomato (Reuscher et al., 2013) have been identified. Information on aquaporin protein accumulation in $S$. melongena is limited. With the development of functional genomics, a large number of expressed sequence tags (ESTs) have been developed for plant species and these sequences are deposited in specific species databases and in GenBank (http://www.ncbi.nlm.nih.gov/ Genbank/index.html) which is publicly available for large scale analysis. As EST-derived microsatellites are from conserved coding sequences of the genome, and they act as functional markers. Several drought responsive genes have been identified and studied by ESTs.

Aquaporin gene expression patterns reveal the role of water channels to root water transport. In-silico analysis of aquaporin genes in S. melongena could be the first step towards revealing the contribution of aquaporins to water and solute transport during crop development. By using bioinformatics techniques with genetic information, known DNA sequences and coded protein sequences to the unknown gene and protein can be compared. Protein domains are often conserved across many species and characterization of these domains has given way to the classification of genes into families, enabling to assign function to similar other genes. As microsatellite markers are involved in gene regulation, locating these markers using conserved protein families approach will be useful in identifying genes associated with them and their specific function. In view of the above, this study was carried out to identify aquaporin markers in response to water stress. In this regard, a computational method was developed to identify these markers from the expressed regions of $S$. melongena.

\section{Materials and Methods}

The EST data available in the public domain for brinjal (Solanum melongena) were downloaded in fasta format from http://www.ncbi.nlm.nih.gov/nucest for identifying markers implicated in stress tolerance. In this study, the first step was to develop a mathematical algorithm based computer program (Thomas et al., 2017) which would identify di, tri, tetra, penta and upto octa repeats from the EST collections. The EST sequences were tagged with identifiers and these EST collections were analyzed for repeat sequences. Subsequently, the EST sequences containing low complexity repeat sequences with an average length of around 500 nucleotides were processed as batch files against NCBI nucleotide database, after removing all the redundant sequences, the sequences of non-redundant ESTs were loaded on NCBI Blast. The identification and clustering of unique EST sequences with motifs was obtained by refining the NCBI BLAST results. From the BLAST results, the matching alignments of similar sequences from Solanaceae were analysed. The ESTs having repeat motif were clustered into groups and analyzed for conserved regions. The sequences having conserved regions along with their IDs were subjected to $\mathrm{NCBI} C D$ search tool for searching the conserved domain database with protein query sequences (Chandraprakash et al., 2019) and generates list of conserved domain hits and their superfamilies description against each protein queries. Finally, the unique $S$. melongena aquaporin sequences were further refined by repeated pair-wise BLAST search. The matching ESTs of Solanum melongena with protein domains and their respective superfamilies were grouped into smaller groups associated with abiotic stress tolerance.

\section{Results and Discussion}

The identified potential EST sequences having markers were compared with published aquaporin proteins. The protein databases available for model plants were used as reference databases for cross comparison and analyzing data obtained from S. melongena. From the results obtained by comparative analysis, 22 sequences viz., FS028035, FS035000, FS042054, FS047611, FS020305, FS022093, FS049874, FS050128, FS050363, FS050393, FS050754, FS050904, FS050980, FS051061, FS051996, FS052264, FS052585, FS052893, FS052964, FS053173, FS000434 and FS051738 were found to be associated with aquaporin proteins. These water channel proteins belong to highly conserved major intrinsic proteins (MIP) superfamily which play an important role in transport of water in plants (Afzal et al., 2016), its regulation and protein expression (Hachez et al., 2006). These aquaporin isoforms with distinct cell type and tissue-specific expression patterns are present in plants where some are constitutively expressed, while others are regulated in response to environmental factors, such as drought and salinity. The identified marker regions present in the ESTs were inherent part of the sequence and associated with aquaporin TIP proteins. These were also found in Solanum nigrum, Solanum lycopersicum, Solanum tuberosum, Capsicum annuum and Nicotiana tomentosiformis.

Tonoplast Intrinsic Proteins (TIPs) are the most abundant aquaporins in the vacuolar membrane (Maurel et al., 2008) and were the first proteins identified for water transporting function (Johnson et al., 1990). Water permeability of the tonoplast is greater than that of the plasma membrane because of highly 


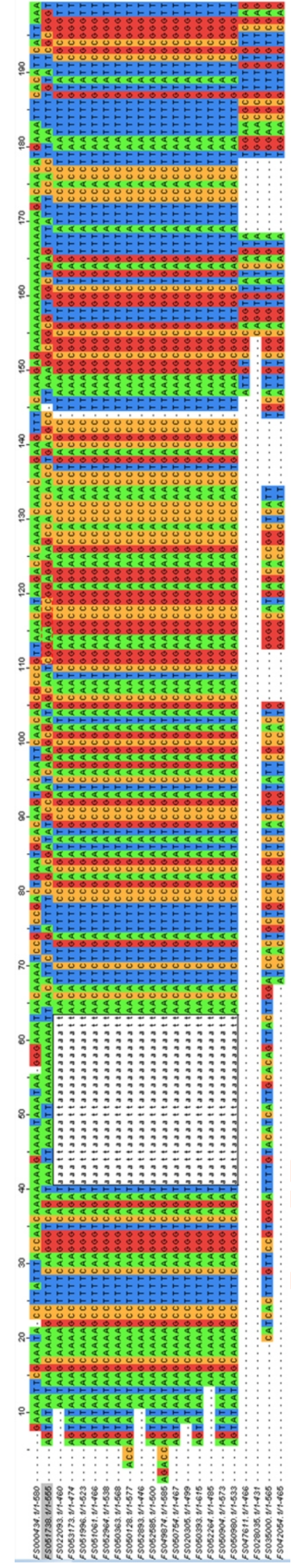

abundant tonoplast aquaporins (Fleurat-Lessard et al., 2005) which thus regulates rapid osmotic adjustment of cytoplasm and maintain turgor pressure of the cell. The average length of matching regions with the identified sequences were found to be $608 \mathrm{bp}$ (Table1). These highly expressed sequences were found to be evolutionarily conserved and identical with published aquaporin proteins of Solanaceous crops viz., chilli (MartínezBallesta et al., 2003), tomato (Balarynová et al., 2018), S. tuberosum etc. (Venkatesh et al., 2013), which facilitate transport of water through cell membranes. It was reported that these expressed regions have been identified in roots of many plant species like Arabidopsis (Postaire et al., 2010), rice (Sakurai et al., 2008), maize (Hachez et al., 2006) and barley (Knipfer et al., 2011), which play a crucial role in plasma membrane, transcellular water transport and TIPs, which show high expression level in roots. These 22 non-redundant ESTs of $S$. melongena with microsatellite markers associated with aquaporin are given below (Table 1) along with EST length, number of similar proteins identified in other species, repeat motif.

Multiple sequence alignment: Further, these EST sequences were aligned using multiple sequence alignment (Fig. 1) for its conserved marker regions within the potential EST sequences. Multiple sequence alignment (MSA) reveals highly conserved region in EST sequences (Bilu et al., 2006). The alignment showed conserved marker region with repeat motif pattern that occur several times within the sequence as shown in (Fig.1).

The marker sequence having 56 bp length was conserved across 17 sequences out of 22 aquaporins of $S$. melongena with a repeat motif pattern AAATTA repeated twice. These sequences

Branch length: $\bullet$ Cladogram $\bigcirc$ Real

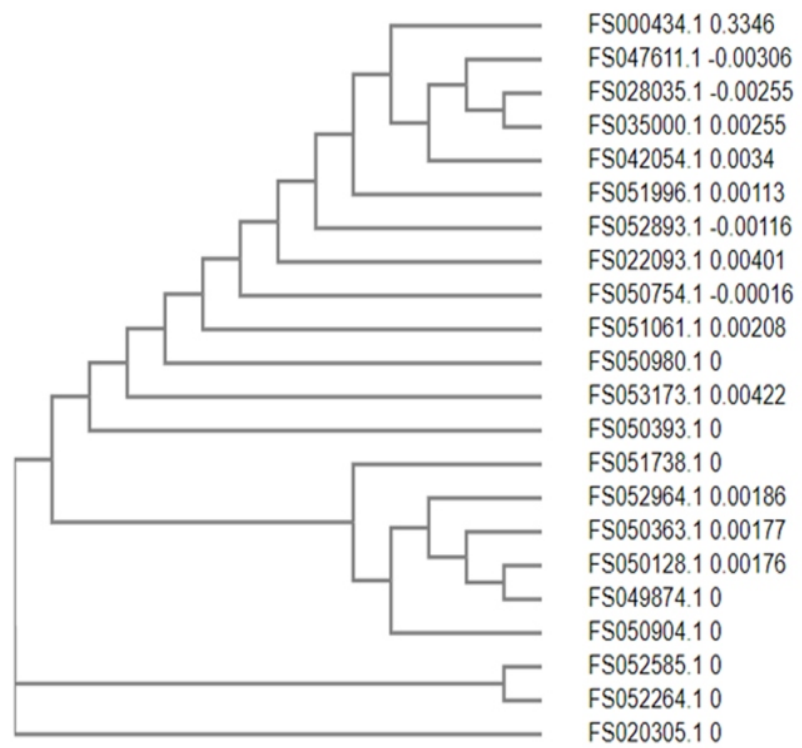

Fig. 2: Phylogenetic tree of aquaporin EST sequences in S. melongena. 
Table 1: ESTs of S. melongena having microsatellite markers associated with MIP superfamily

\begin{tabular}{|c|c|c|c|c|}
\hline $\begin{array}{l}\text { Brinjal- } \\
\text { EST Id }\end{array}$ & $\begin{array}{l}\text { EST } \\
\text { length }\end{array}$ & $\begin{array}{l}\text { Motif } \\
\text { pattern }\end{array}$ & $\begin{array}{l}\text { Matches with } \\
\text { published } \\
\text { proteins }\end{array}$ & Species \\
\hline Fs000434 & 580 & $(\mathrm{AAAC})_{2}$ & 4 & Capsicum annuum, Solanum nigrum, Solanum lycopersicum, Solanum tuberosum \\
\hline Fs020305 & 499 & $(\text { AAATTA })_{2}$ & 2 & Solanum lycopersicum, Solanum tuberosum \\
\hline Fs022093 & 460 & $(\text { AAATTA })_{2}$ & 2 & Solanum lycopersicum, Solanum tuberosum \\
\hline Fs028035 & 431 & $(\mathrm{CTGGAC})_{2}$ & 4 & Capsicum annuum, Solanum nigrum, Solanum lycopersicum, Solanum tuberosum \\
\hline Fs035000 & 565 & $(\mathrm{CTGGAC})_{2}$ & 4 & Capsicum annuum, Solanum nigrum, Solanum lycopersicum, Solanum tuberosum \\
\hline Fs042054 & 465 & $(\mathrm{CTGGAC})_{2}$ & 4 & Capsicum annuum, Solanum nigrum, Solanum lycopersicum, Solanum tuberosum \\
\hline Fs047611 & 466 & $(\text { CTGGAC })_{2}$ & 4 & Capsicum annuum, Solanum nigrum, Solanum lycopersicum, Solanum tuberosum \\
\hline Fs049874 & 585 & $(\mathrm{AAATTA})_{2}$ & 3 & Solanum lycopersicum, Solanum tuberosum, Nicotianatomentosiformis \\
\hline Fs050128 & 577 & $(\text { AAATTA })_{2}$ & 3 & Solanum lycopersicum, Solanum tuberosum, Nicotianatomentosiformis \\
\hline Fs050363 & 568 & $(\text { AAATTA })_{2}$ & 2 & Solanum lycopersicum, Solanum tuberosum \\
\hline Fs050393 & 615 & $(\mathrm{AAATTA})_{2}$ & 3 & Solanum lycopersicum, Solanum tuberosum, Nicotianatomentosiformis \\
\hline Fs050754 & 467 & $(\text { AAATTA })_{2}$ & 2 & Solanum lycopersicum, Solanum tuberosum \\
\hline Fs050904 & 573 & $(\text { AAATTA })_{2}$ & 3 & Solanum lycopersicum, Solanum tuberosum, Nicotianatomentosiformis \\
\hline Fs050980 & 533 & $(\text { AAATTA })_{2}$ & 3 & Solanum lycopersicum, Solanum tuberosum, Nicotianatomentosiformis \\
\hline Fs051061 & 466 & $(\text { AAATTA })_{2}$ & 2 & Solanum lycopersicum, Solanum tuberosum \\
\hline Fs051738 & 555 & $(\text { AAATTA })_{2}$ & 3 & Solanum lycopersicum, Solanum tuberosum, Nicotianatomentosiformis \\
\hline Fs051996 & 523 & $(\text { AAATTA })_{2}$ & 3 & Solanum lycopersicum, Solanum tuberosum, Nicotianatomentosiformis \\
\hline Fs052264 & 485 & $(\text { AAATTA })_{2}$ & 2 & Solanum lycopersicum, Solanum tuberosum \\
\hline Fs052585 & 500 & $(\text { AAATTA })_{2}$ & 2 & Solanum lycopersicum, Solanum tuberosum \\
\hline Fs052893 & 446 & $(\text { AAATTA })_{2}$ & 1 & Solanum tuberosum \\
\hline Fs052964 & 538 & $(\text { AAATTA })_{2}$ & 3 & Solanum lycopersicum, Solanum tuberosum, Nicotianatomentosiformis \\
\hline Fs053173 & 474 & $(\text { AAATTA })_{2}$ & 2 & Solanum lycopersicum, Solanum tuberosum \\
\hline
\end{tabular}

remain evolutionarily conserved with microsatellite markers, shown in white color (Fig.1). The alignments of 22 sequences revealed the robustness of these microsatellite markers in the aquaporin genes coding regions. The conserved consensus motifs exist across the aquaporin subfamilies to support their association.

Phylogenetic tree: The alignments of sequence were also performed by using the default parameter of Clustal W program (Tamura et al., 2013) and a phylogenetic tree was constructed (Fig.2) as per Neighbor-Joining tree method. The phylogenetic analysis revealed that these 22 sequences show the evolutionarily-conservedness of multigene aquaporin family present in several species of Solanaceae such as Solanum nigrum, Solanum lycopersicum, Solanum tuberosum, Capsicum annuum, Nicotiana tomentosiformis as aquaporin TIP protein are present in most of them. However, the development of markers from conventional method is timeconsuming and expensive due to sequencing requirements from several sources. Here, we found unique aquaporin markers within available EST collections, by computational method using repeat motif identifier. In doing so, we identified potential aquaporin markers present in 22 expressed regions from 98089 EST collections of brinjal. These markers would be useful in plant breeding program for development of water stress tolerant crops to regulate water uptake by roots during water deficit conditions.

\section{Acknowledgments}

The project was funded by ICAR-Centre for Agricultural Bioinformatics project and the authors wish to thank the PI of Network project on Agricultural Bioinformatics and Computational Biology, ICAR-IASRI.

\section{References}

Afzal, Z., T. Howton, Y. Sun and M. Mukhtar: The roles of aquaporins in plant stress responses. J. Dev. Biol., 9, 1-22 (2016).

Balarynová, J., J. Danihlík and M. Fellner: Changes in plasma membrane aquaporin gene expression under osmotic stress and blue light in tomato. Acta Physiol. Plant., 40, 27-34 (2018).

Barrieu, F., F. Chaumont and M.J. Chrispeels: High expression of the tonoplast aquaporin Zm TIP1 in epidermal and conducting tissues of maize. Plant Physiol., 117, 1153-1163(1998).

Behboudian, M.H.: Responses of eggplant to drought. I. Plant water balance. Sci. Hort., 7, 303-310 (1977).

Bilu, Y., P.K. Agarwal and R. Kolodny: Faster algorithms for optimal multiple sequence alignment based on pairwise comparisons. IEEE/ACM Transactions on Computational Biology and Bioinformatics, 3, 408-422 (2006). 
ChandraPrakash, M.K. and R.R. Thomas: Genome wide identification of nucleotide-binding site leucine-rich repeat (NBS-LRR) gene encoding regions in Solanum lycopersicum resistant to environmental pathogens by computational methods. J. Environ. Biol., 40, 613-618 (2019).

Chaumont, F., F. Barrieu, E. Wojcik, M.J. Chrispeels and R. Jung: Aquaporins constitute a large and highly divergent protein family in maize. Plant Physiol., 125, 1206-1215 (2001).

Chrispeels, M.J., N.M. Crawford and J.I. Schroeder: Proteins for transport of water and mineral nutrients across the membranes of plant cells. Plant Cell, 11, 661-675 (1999).

Cushman, J.C. and H.J. Bohnert: Genomic approaches to plant stress tolerance. Curr. Opin. Plant Biol., 3, 117-124 (2000).

Fleurat-Lessard, P., P. Michonneau, M. Maeshima, J.J. Drevon and R. Serraj: The distribution of aquaporin subtypes (PIP1, PIP2 and y$\mathrm{TIP}$ ) is tissue dependent in soybean (Glycine max) root nodules. Ann. Bot., 96, 457-460 (2005)

Hachez, C., M. Moshelion, E. Zelazny, D. Cavez and F. Chaumont: Localization and quantification of plasma membrane aquaporin expression in maize primary root: Aclue to understanding their role as cellular plumbers. Plant Mol. Biol., 62, 305-323 (2006).

Hirakawa, H., K. Shirasawa, K. Miyatake, T. Nunome, S. Negoro, A. Ohyama and $\mathrm{H}$. Fukuoka: Draft genome sequence of eggplant (Solanum melongena L.): The representative Solanum species indigenous to the Old World. DNARes., 21, 649-60 (2014).

Johanson, U., M. Karlsson, I. Johansson, S. Gustavsson, S. Sjövall, L. Fraysse, A.R. Weig and P. Kjellbom: The complete set of genes encoding major intrinsic proteins in Arabidopsis provides a framework for a new nomenclature for major intrinsic proteins in plants. Plant Physiol., 126,1358-1369 (2001).

Johansson, I., M. Karlsson, U. Johanson, C. Larsson and P. Kjellbom: The role of aquaporins in cellular and whole plant water balance. Biochim. Biophys. Acta., 1465, 324-342 (2000).

Johnson, K.D., H. Höfte and M.J. Chrispeels: An intrinsic tonoplast protein of protein storage vacuoles in seeds is structurally related to a bacterial solute transporter (GlpF). Plant Cell, 2, 525-532 (1990).

Kapilan, R., V. Maryam and J.J. Zwiazek: Regulation of aquaporins in plants under stress. Biol. Res., 51, 4 (2018).

Knipfer, T., M. Besse, J.L. Verdeil and W. Fricke: Aquaporin-facilitated water uptake in barley (Hordeum vulgare L.) roots. J. Exp. Bot., 62, 4115-4126 (2011).
Martínez-Ballesta, M.C., V. Martínez and M. Carvajal: Aquaporin functionality in relation to H+-ATPase activity in root cells of Capsicum annuum, L. grown under salinity. Physiol. Plant., 117, 413-420 (2003).

Maurel, C., L. Verdoucq, D.T. Luu and V. Santoni: Plant aquaporins: Membrane channels with multiple integrated functions. Annu. Rev. Plant Biol., 59, 595-624 (2008).

Postaire, O., C. Tournaire-Roux, A. Grondin, Y. Boursiac, R. Morillon, T. Schäffner and C. Maurel: A PIP1 aquaporin contributes to hydrostatic pressure-induced water transport in both the root and rosette of Arabidopsis. Plant Physiol., 152, 1418-1430 (2010).

Preston, G.M., T.P. Carroll, W.B. Guggino and P. Agree: Appearance of water channels in Xenopus oocytes expressing red cell CHIP 28 proteins. Science, 256, 385-387(1992).

Reuscher, S., M. Akiyama, C. Mori, K. Aoki, D. Shibata and K. Shiratake: Genome-wide identification and expression analysis of aquaporins in tomato. PLoS ONE, 8, e79052 (2013).

Sakurai, J., F. Ishikawa, T. Yamaguchi, M. Uemura and M. Maeshima: Identification of 33 rice aquaporin genes and analysis of their expression and function. Plant Cell Physiol., 46, 1568-1577 (2005).

Sakurai, J., A. Ahamed, M. Murai, M. Maeshima and M. Uemura: Tissue and cell-specific localization of rice aquaporins and their water transport activities. Plant Cell Physiol., 49, 30-39 (2008).

Tamura, K., G. Stecher, D. Peterson, A. Filipski and S. Kumar: MEGA6: Molecular evolutionary genetics analysis. Mol. Biol. Evol., 30, 2725-2729 (2013).

Thomas, R.R., M.K. Chandraprakash, M.K. Reddy, R. Mahmood and P. Mondal: EST linked markers in Solanum melongena responsive to abiotic stress using computational methods. The Ecoscan, 11-12, 45-49 (2017).

Tyerman, S.D., H.J. Bohnert, C. Maurel, E. Steudle and J.A.C. Smith: Plant aquaporins: Their molecular biology, biophysics and significance for plantwater relations. J. Exp. Bot., 50, 1055-1071 (1999).

Venkatesh, J., J.W. Yu and S.W. Park: Genome-wide analysis and expression profiling of the Solanum tuberosum aquaporins. Plant Physiol. Biochem., 73, 392-404 (2013).

Zhang, D.Y., Z. Ali, C.B. Wang, L. Xu, J.X. Yi, Z.L. Xu, X.Q. Liu, X.L. He, Y.H. Huang, I.A. Khan, R.M. Trethowan and H.X. Ma: Genomewide sequence characterization and expression analysis of major intrinsic proteins in soybean (Glycine max L.). PLOS ONE, 8, e56312(2013). 